\title{
SPIRE imaging of M 82: Cool dust in the wind and tidal streams ${ }^{\star}$
}

\author{
H. Roussel ${ }^{1}$, C. D. Wilson ${ }^{2}$, L. Vigroux ${ }^{1}$, K. G. Isaak ${ }^{3,4}$, M. Sauvage ${ }^{5}$, S. C. Madden ${ }^{5}$, R. Auld ${ }^{3}$, M. Baes ${ }^{6}$, \\ M. J. Barlow ${ }^{7}$, G. J. Bendo ${ }^{8}$, J. J. Bock ${ }^{9}$, A. Boselli ${ }^{10}$, M. Bradford ${ }^{9}$, V. Buat ${ }^{10}$, N. Castro-Rodriguez ${ }^{11}$, P. Chanial ${ }^{5}$, \\ S. Charlot ${ }^{1}$, L. Ciesla ${ }^{10}$, D. L. Clements ${ }^{8}$, A. Cooray ${ }^{12}$, D. Cormier ${ }^{5}$, L. Cortese ${ }^{3}$, J. I. Davies ${ }^{3}$, E. Dwek ${ }^{13}$, \\ S. A. Eales ${ }^{3}$, D. Elbaz ${ }^{5}$, M. Galametz ${ }^{5}$, F. Galliano ${ }^{5}$, W. K. Gear ${ }^{3}$, J. Glenn ${ }^{14}$, H. L. Gomez ${ }^{3}$, M. Griffin ${ }^{3}$, S. Hony ${ }^{5}$, \\ L. R. Levenson ${ }^{9}$, N. Lu ${ }^{9}$, B. O'Halloran ${ }^{8}$, K. Okumura ${ }^{5}$, S. Oliver ${ }^{15}$, M. J. Page ${ }^{16}$, P. Panuzzo ${ }^{5}$, A. Papageorgiou ${ }^{3}$, \\ T. J. Parkin ${ }^{2}$, I. Perez-Fournon ${ }^{11}$, M. Pohlen ${ }^{3}$, N. Rangwala ${ }^{14}$, E. E. Rigby ${ }^{17}$, A. Rykala ${ }^{3}$, N. Sacchi ${ }^{18}$, B. Schulz ${ }^{19}$, \\ M. R. P. Schirm ${ }^{2}$, M. W. L. Smith ${ }^{3}$, L. Spinoglio ${ }^{18}$, J. A. Stevens ${ }^{20}$, S. Srinivasan ${ }^{1}$, M. Symeonidis ${ }^{16}$, M. Trichas ${ }^{8}$, \\ M. Vaccari ${ }^{21}$, H. Wozniak ${ }^{22}$, G. S. Wright ${ }^{23}$, and W. W. Zeilinger ${ }^{24}$
}

(Affiliations are available in the online edition)

Received 30 March 2010 / Accepted 13 April 2010

\section{ABSTRACT}

M 82 is a unique representative of a whole class of galaxies, starbursts with superwinds, in the Very Nearby Galaxy Survey with Herschel. In addition, its interaction with the M 81 group has stripped a significant portion of its interstellar medium from its disk. SPIRE maps now afford better characterization of the far-infrared emission from cool dust outside the disk, and sketch a far more complete picture of its mass distribution and energetics than previously possible. They show emission coincident in projection with the starburst wind and in a large halo, much more extended than the PAH band emission seen with Spitzer. Some complex substructures coincide with the brightest PAH filaments, and others with tidal streams seen in atomic hydrogen. We subtract the far-infrared emission of the starburst and underlying disk from the maps, and derive spatially-resolved far-infrared colors for the wind and halo. We interpret the results in terms of dust mass, dust temperature, and global physical conditions. In particular, we examine variations in the dust physical properties as a function of distance from the center and the wind polar axis, and conclude that more than two thirds of the extraplanar dust has been removed by tidal interaction, and not entrained by the starburst wind.

Key words. dust, extinction - evolution - galaxies: interactions - galaxies: starburst - infrared: ISM

\section{Introduction}

M 82 is the closest specimen of starbursts with superwinds, at a distance of only (3.9 \pm 0.3$)$ Mpc (Sakai \& Madore 1999). Its almost edge-on orientation is ideal for the study of the superwind, and more generally the interaction of galaxies with the intergalactic medium and the role of the gas and dust dynamics in the self-regulation of starbursts.

The exceptional star formation activity of M 82 is thought to be caused by its tidal interaction with the M 81 galaxy group, redistributing the gas within the disk of M 82 toward the central regions where it accumulates, and expelling atomic gas in giant streams connecting the disk to the intragalactic medium (Yun et al. 1994). M 82 is an evolved starburst, in the sense that its activity has been sustained (episodically) over much of its lifetime. Coupled stellar population synthesis and photoionization models reveal two major episodes of star formation over the past $10 \mathrm{Myr}$ (Förster Schreiber et al. 2003). Numerous young supernova remnants have been detected (Muxlow et al. 1994). Another peak of star formation about $150 \mathrm{Myr}$ ago is inferred from the age-dating of stellar clusters (Konstantopoulos et al. 2009), and 200 super star clusters, signposts of starbursts, have been found in M 82 (Melo et al. 2005), spanning a wide age interval from $60 \mathrm{Myr}$ to several Gyr (Gallagher \& Smith 1999; de Grijs et al. 2001).

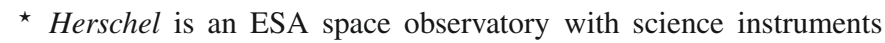
provided by European-led Principal Investigator consortia, and with important participation from NASA.
Ward et al. (2003) found that more than half the total molecular gas of the central regions is warmer than $50 \mathrm{~K}$, and Mao et al. (2000) found that CO transitions up to $J=7-6$ trace diffuse gas abundantly illuminated by UV radiation. Panuzzo et al. (2010) modeled CO transitions from $J=4-3$ to $J=13-12$ in the central $43^{\prime \prime}$, observed with the SPIRE FTS spectrometer, and they suggest that turbulent heating caused by stellar winds and supernovae may be the dominant excitation process for the highest transitions, tracing gas at about $500 \mathrm{~K}$. The molecular gas properties thus reinforce the notion that M 82 is an evolved starburst, which has been active for long enough to completely disrupt the cold component of the interstellar medium.

The mechanical energy simultaneously released by a large collection of massive stars and supernovae is able to drive a superwind detected in both X-rays emitted by shocked gas (Strickland et al. 1997) and optical lines excited by a combination of photoionization and shock ionization (Shopbell \& Bland-Hawthorn 1998). Optical emission around the wind also partly issues from a reflection nebula (Scarrott et al. 1991), indicating the presence of dust. Abundant molecular gas is associated with both the superwind (although probably not spatially coincident but rather outside the bicylindrical outflow) and tidal streams (Walter et al. 2002), and PAH emission is found in a very extended halo (Engelbracht et al. 2006).

This paper studies the spatially-resolved properties of the cool dust detected by SPIRE. Since PACS photometric observations had not been scheduled at the time of writing, and Spitzer 
MIPS images are saturated, we sample the far-infrared spectral energy distribution only longward of $250 \mu \mathrm{m}$, where it can be safely assumed that the emission is dominated by grains in thermal equilibrium. SCUBA maps have higher angular resolutions than SPIRE maps; their sensitivities are however too low to reliably detect any emission outside the disk of M 82, even when all archival data are combined (Leeuw \& Robson 2009). In contrast, bright filaments and diffuse emission are detected out to very large distances with SPIRE. We thus focus on the dust content and physical properties outside the main body of M 82, where SPIRE maps provide truly unique information.

\section{SPIRE observations, data reduction, and analysis}

M 82 was observed with the SPIRE photometer arrays onboard Herschel (Pilbratt et al. 2010) during the science demonstration phase. The SPIRE instrument, its in-orbit performance, and scientific capabilities are described by Griffin et al. (2010). Its resolving power enables a linear resolution of 350 to $700 \mathrm{pc}$ in M 82, from 250 to $500 \mu \mathrm{m}$ ( $1 \mathrm{kpc}$ corresponds to $\left.53^{\prime \prime}\right)$.

SPIRE maps of $22^{\prime} \times 22^{\prime}$ were acquired in nominal scan mode, with a total of 4 scans. The data were processed with HIPE 3.0 up to level 1, as described by Pohlen et al. (2010), except without the thermal drift correction. The data were then input to the Scanamorphos software (Roussel, in preparation), which produces maps after subtracting the thermal drift and the low-frequency noise of each bolometer, purely by using the available redundancy. The resulting maps have $\sim 30 \%$ smaller sky standard deviations and $\sim 3 \%$ larger beam areas.

We also derived SPIRE point response functions (PRF) ${ }^{1}$ from a nominal 8-scan observation of Uranus, with a field of view of $20^{\prime} \times 20^{\prime}$, that we processed in the same way and with the same orientation along the first $\mathrm{scan}^{2}$. This observation is deeper than that of Neptune - made in bright-source mode used for the official PRF release, and thus allows detection of the secondary lobes and diffraction spikes. Uranus is slightly more extended than Neptune (3.53" at the time of observation ${ }^{3}$, as opposed to $2.26^{\prime \prime}$ ), but suitable for sources larger than $70 \mathrm{pc}$ in M 82. To create the PRF, all the emission outside the beam pattern was masked out, and the few remaining background sources were approximated by Gaussians and subtracted.

All maps have a pixel size of $4.5^{\prime \prime}$ at $250 \mu \mathrm{m}, 6.25^{\prime \prime}$ at $350 \mu \mathrm{m}$, and $9^{\prime \prime}$ at $500 \mu \mathrm{m}$, i.e. approximately one fourth of the beam full width at half maximum $(F W H M)$.

Photometry: Since the flux calibration of SPIRE is strictly valid for point sources only, we used our empirical beams (corrected for the finite size of Uranus) to estimate beam areas of 468 , 850 , and 1763 square arcseconds, respectively at 250, 350, and $500 \mu \mathrm{m}$, and obtain extended-source flux calibration. They are larger than the current official estimates (based on Gaussian fits, i.e. not including sidelobes and spikes) by 10 to $20 \%$.

We measure global flux densities of $(457 . \pm 2$.) Jy at $250 \mu \mathrm{m}$, $(155 . \pm 2$.) Jy at $350 \mu \mathrm{m}$, and (49.6 \pm 0.9$) \mathrm{Jy}$ at $500 \mu \mathrm{m}$ (error bars not including systematic flux calibration uncertainties of $15 \%$,

\footnotetext{
1 This term designates the result of sampling the point spread function (PSF) of the optics by the combination of the observation pattern and the map pixel grid: it is an empirical realization of the PSF.

2 Files available at http://www2.iap.fr/users/roussel/ spirebeams/.

${ }^{3}$ Using the HORIZONS ephemeris service accessible at http:// ssd.jpl.nasa.gov/horizons.cgi.
}

nor beam size uncertainties). They were multiplied by the recommended corrective factors (see Pohlen et al. 2010).

Source decomposition: The central regions of M 82 have a complex structure. They contain multiple bright infrared sources that can be most easily separated in mid-infrared images (Telesco \& Gezari 1992; Engelbracht et al. 2006). The surface brightness dynamic range between the starburst and the halo emission spans nearly three decades. Therefore, to subtract the central sources and obtain maps of the superwind and halo as minimally contaminated by the diffraction pattern of the bright center as possible, we adapted the CLEAN algorithm used in radio astronomy (Högbom 1974) to substitute "clean" beams for beams with extended sidelobes. At each iteration, the peak of the map is found, and the scaled PRF at the same location is multiplied by a small gain and subtracted from the map; a narrower Gaussian with the same flux is added to a model map. The residual maps obtained after "cleaning" are shown in Fig. 1.

The starburst emission that we recover from the beam "cleaning" process, after adding back to the model maps the underlying residuals (that account for about $7 \%$ of the total), amounts to 337,111 , and $35.4 \mathrm{Jy}$ at 250,350 , and $500 \mu \mathrm{m}$, respectively, i.e. between $70 \%$ and $75 \%$ of the global emission. If we instead sum the emission from the four brightest sources selected from the $350 \mu \mathrm{m}$ SCUBA map, fitted with fixed positions and widths $(\sim 150 \mathrm{pc})$ in all maps, then we already account for about $57 \%$ of the global emission in each SPIRE band.

Since there is a smooth transition from the disk to the offdisk emission, the spatial separation between the two is somewhat arbitrary. We note that the disk of M 82 is very thick and turbulent, and that the residual maps are still contaminated by disk emission. We thus masked pixels where the original flux density exceeds the cleaning threshold, as shown in Fig. 1.

Dust opacities and emissivities: In all the following, we adopt the Galactic emissivity law derived by Reach et al. (1995) from the COBE survey. Dust grain opacities vary as a function of environment and are very uncertain. However, dust masses can easily be rescaled if another value is assumed. We thus adopt an opacity valid for dust in the diffuse interstellar medium, $\kappa=1.62 \mathrm{~m}^{2} \mathrm{~kg}^{-1}$ in the DIRBE $140 \mu \mathrm{m}$ band (Li \& Draine 2001). Converting it to an opacity at $500 \mu \mathrm{m}$, we obtain $0.18 \mathrm{~m}^{2} \mathrm{~kg}^{-1}$, in good agreement with what would have been obtained from the $850 \mu \mathrm{m}$ opacity of James et al. (2002), i.e. $(0.20 \pm 0.06) \mathrm{m}^{2} \mathrm{~kg}^{-1}$.

\section{The wind and tidal streams}

Cool dust is detected at $5 \sigma$ significance in filaments out to $9 \mathrm{kpc}$ from the center, at $250 \mu \mathrm{m}$. The maximal extensions are to the north-east and to the south-east of the center, i.e. in filaments making a large angle with the polar axes of the galaxy and outflow. As already remarked by Engelbracht et al. (2006) for the PAH emission, dust occupies a very extended halo, and is not confined to the edges of what is traditionally called the superwind. Comparison with the HI map of Yun et al. (1994) shows that the densest parts of the tidal streams are associated with some of the extended dust detected in SPIRE maps. Below, we provide quantitative arguments to support the impression that most of the dust mass outside the disk of M 82 has been removed by tidal interaction, rather than entrained by the superwind.

To approximately match the angular resolution of the maps and examine spatial variations in dust colors of the wind and halo, we convolved the "cleaned" maps with Gaussian kernels 

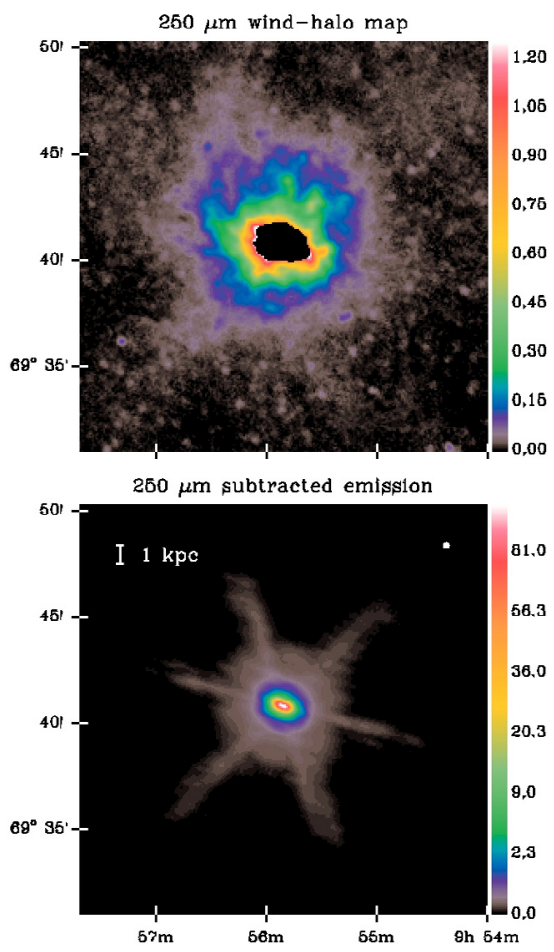

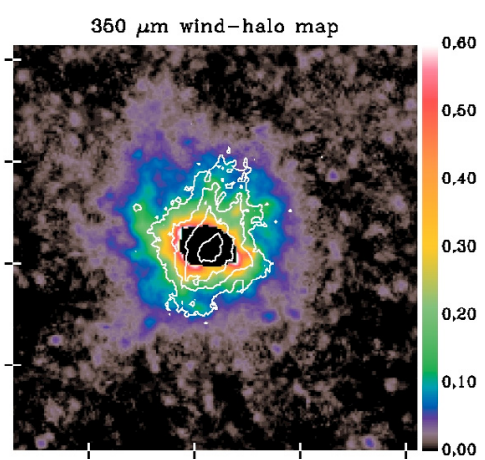

$350 \mu \mathrm{m}$ subtracted emission

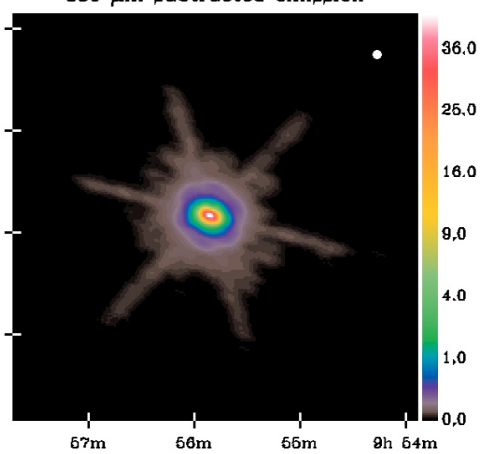

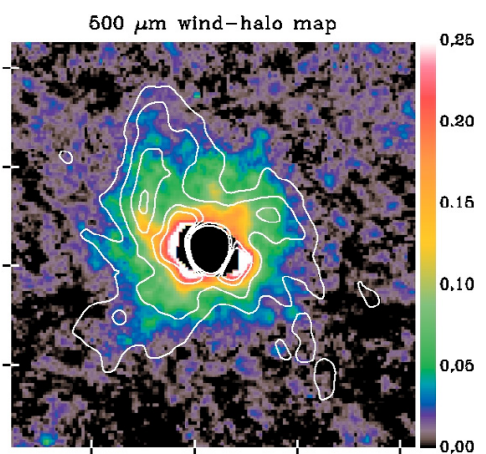

$500 \mu \mathrm{m}$ subtracted emission

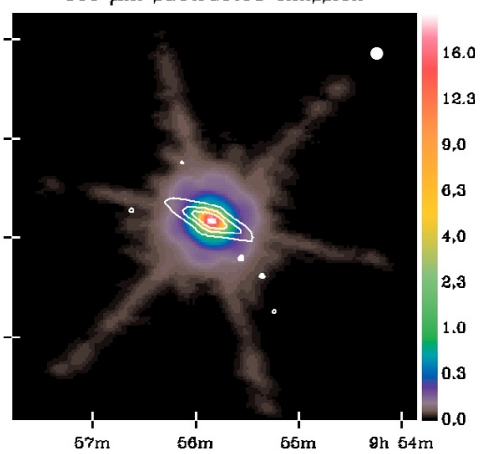

Fig. 1. SPIRE maps resulting from the "cleaning" process. Top row: residual maps showing the wind and halo emission. Contours drawn from the HI map of Yun et al. (1994) and from the H $\alpha$-[NII] map of Boselli \& Gavazzi (2002) are superimposed on the $500 \mu \mathrm{m}$ and $350 \mu \mathrm{m}$ maps, respectively. Bottom row: subtracted central emission. The stellar disk is delineated by the IRAC $3.6 \mu \mathrm{m}$ contours on the $500 \mu \mathrm{m}$ map. The initial maps have peak brightnesses of 105, 48.2, and $21.6 \mathrm{Jy} / \mathrm{beam}$, at 250,350, and $500 \mu \mathrm{m}$ respectively. The beam FWHM is sketched to the top right.
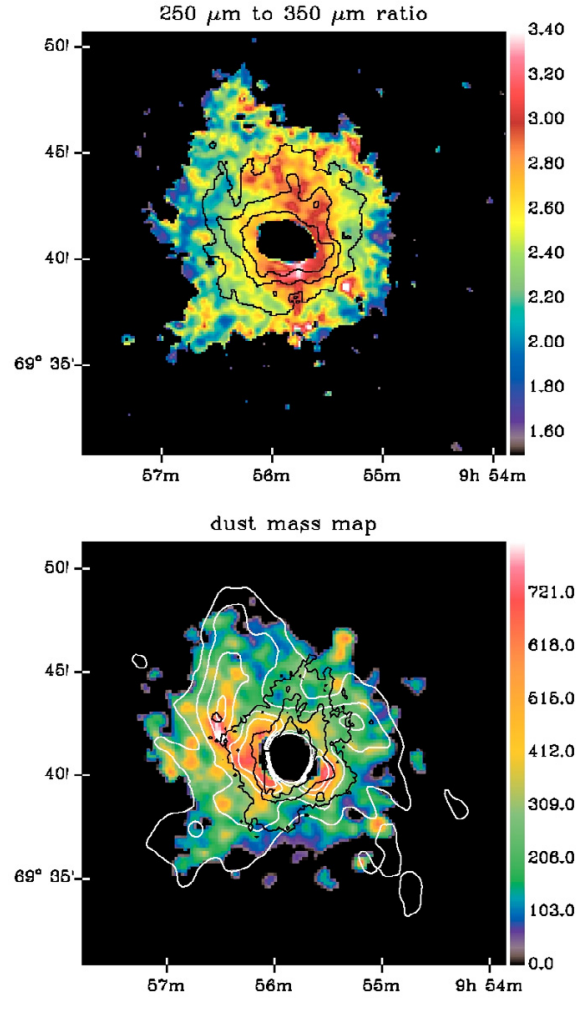

$250 \mu \mathrm{m}$ to $500 \mu \mathrm{m}$ ratio

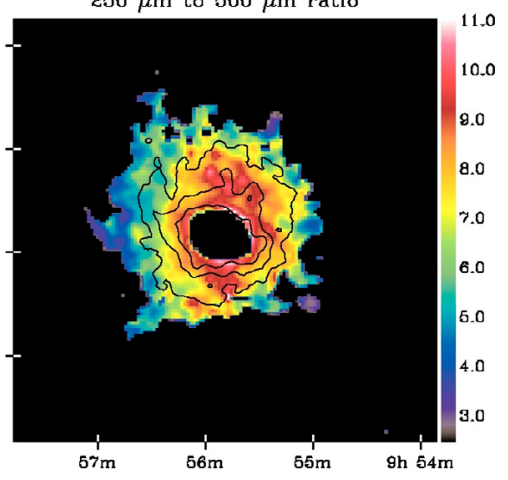

$6 \mu \mathrm{m}$ to $250 \mu \mathrm{m}$ ratio

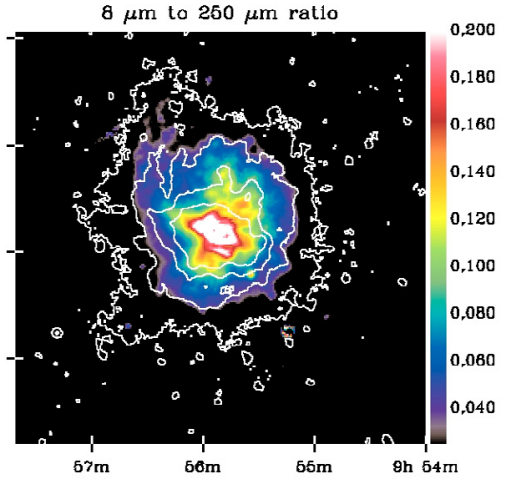

Fig. 2. Top row: flux ratio maps. Left: $250 \mu \mathrm{m}$ to $350 \mu \mathrm{m}$ ratio, at $28^{\prime \prime}$ angular resolution (FWHM). Right: $250 \mu \mathrm{m}$ to $500 \mu \mathrm{m}$ ratio, at $40^{\prime \prime}$ angular resolution $(F W H M)$. Contours of the $250 \mu \mathrm{m}$ map are overlaid in black. Bottom row: Left: dust mass map in $M_{\odot}$ per pixel $(F W H M$ of $40^{\prime \prime}$, corresponding to $760 \mathrm{pc}$; pixels are $170 \mathrm{pc}$ on a side). HI contours are superimposed in white and $\mathrm{H} \alpha$ contours in black. Special note: the dust blob located around 09:55:23 $+69: 46: 10$ is likely associated with M 82, rather than a background source, since its morphology in all SPIRE bands is diffuse. Right: $8 \mu \mathrm{m}$ to $250 \mu \mathrm{m}$ flux ratio map of the wind and halo, at the $250 \mu \mathrm{m}$ resolution. Contours of the $250 \mu \mathrm{m}$ map are overlaid. of appropriate widths, and regridded the resulting maps to the pixel size at the longest wavelength. Figure 2 shows the $250 \mu \mathrm{m}$ to $350 \mu \mathrm{m}$ ratio map and the $250 \mu \mathrm{m}$ to $500 \mu \mathrm{m}$ ratio map. Based on these ratios, the dust temperature in the wind and halo ranges between about $12 \mathrm{~K}$ and $50 \mathrm{~K}$, and its average value decreases with projected distance from the starburst. Some filaments that are much warmer than their surroundings can be seen; they are located in-between bright dust spurs (Fig. 2), and thus must have relatively low densities. The present data do not allow us to test whether these color variations are caused purely by different excitation conditions, or whether they are affected by changes in the dust composition within the wind. Assuming that these 
filaments follow the Galactic emissivity law, we estimate that they have temperatures between $30 \mathrm{~K}$ and $50 \mathrm{~K}$, i.e. at least as high as in the starburst. They seem to be associated with the starburst wind, rather than with the tidal features traced by the HI map.

We estimate the total dust mass in the wind and halo from the $500 \mu \mathrm{m}$ and dust temperature maps, the latter derived from the $250 \mu \mathrm{m}$ to $500 \mu \mathrm{m}$ ratio. We obtain $M_{\mathrm{d} \text { halo }}=(1.1 \pm 0.5) \times 10^{6} M_{\odot}$ (mass error map summed linearly, and $2.5 \sigma$ cut applied to the brightness maps). The mass derived from the global halo fluxes and a single-temperature fit (with $T_{\text {dust }}=22.3$ to $25.6 \mathrm{~K}$ ) is similar: $M_{\mathrm{d} \text { halo }}=(1.0$ to 1.2$) \times 10^{6} M_{\odot}$. This is much lower than the SCUBA estimate of Alton et al. (1999) ( 2 to $\left.10 \times 10^{6} M_{\odot}\right)$, even using the same dust opacity. We cannot rule out a cold component undetected by SPIRE, but the discrepancy may predominantly arise from several analysis issues: although the halo is barely detected in the SCUBA maps (because they are only $2.6 \mathrm{kpc}$ in diameter, and the chop throw placed the sky reference within the wind), the starburst and disk have not been subtracted with their sidelobes, and the halo emission may thus be severely contaminated by them; the procedure followed by Alton et al. (1999) to estimate the dust temperature also entails large errors.

The combination of the dust temperature and brightness maps shows that dust that is spatially coincident with the superwind (likely to have been entrained by it) is warmer and more diffuse than dust that is associated with the tidal streams, the latter consequently dominant in terms of mass, as illustrated by the dust mass map in Fig. 2. Defining large sectors to exclude the superwind regions, based on $\mathrm{H} \alpha$ and $\mathrm{X}$-ray maps (with opening angles of $74^{\circ}$ in the north and $64^{\circ}$ in the south), we argue that at a minimum $65 \%$ of the halo dust mass is not associated with the superwind. Since tidal features overlap in projection with superwind features, this fraction is a strict lower limit.

The global dust mass of M 82, folding in the $1.25 \mathrm{~mm}$ measurement of Thuma et al. (2000), is $M_{\mathrm{d} \text { tot }} \sim(4.5 \pm 1.4) \times 10^{6} M_{\odot}$ (with $T_{\text {dust }}=24 \mathrm{~K}$, which provides a fit too low by only $3 \%$ at $350 \mu \mathrm{m}$ and by $22 \%$ at $250 \mu \mathrm{m}$ ). If our mass estimates are correct, they imply that $\sim 25 \%$ of all the dust has been expelled from the disk. Either the efficiency of gas and dust ejection by the superwind and the tidal interaction is very high, or the dust grain composition and size distribution in the wind and halo significantly differ from those in the starburst. Without data of comparable quality at both shorter and longer wavelengths, we cannot test the second alternative, but we deem it less likely.

Since the interstellar medium of M 82 must have become extremely turbulent and porous as a result of the sustained injection of mechanical energy by the successive starbursts, probably enough ultraviolet radiation is leaking out of the disk to provide most of the dust heating necessary to account for the observed emission. GALEX images show diffuse far-UV and near-UV light scattered by dust grains out to large distances (Hoopes et al. 2005), at least up to $6 \mathrm{kpc}$ on the southern side. However, this light is mainly confined to lobes around the minor axis, whereas dust emission is not. Optical photons must dominate the heating further away from the minor axis.

Using the archival Spitzer $8 \mu \mathrm{m}$ image to trace PAHs in the wind (Engelbracht et al. 2006), we find that the PAH emission does not extend as far out as the $250-500 \mu \mathrm{m}$ emission, and that there is a steep radial gradient in the $8 \mu \mathrm{m}$ to $250 \mu \mathrm{m}$ flux ratio (Fig. 2). There are also some regions of enhanced PAH brightness relatively close to the polar axis, which correspond well to the $250 \mu \mathrm{m}$ spurs coincident with $\mathrm{H} \alpha$ emission. These regions contain relatively warm dust. We hypothesize that they are well outside the wind shock, where PAHs would have very short lifetimes (Micelotta et al. 2010), and that they simply correspond to more efficient illumination by UV photons leaking through discrete cavities in the disk. A large fraction of optical line emission in this region is also due to photoionization, and not only to shocks (Shopbell \& Bland-Hawthorn 1998). The general gradient in the $8 \mu \mathrm{m}$ to $250 \mu \mathrm{m}$ flux ratio and in the far-infrared flux ratios would then be a natural consequence of the dilution of the radiation field with distance from the emitting stars.

\section{By way of conclusion}

The intergalactic medium can be enriched in metals by both tidal stripping and starburst winds. M 82 offers an exquisite example of both processes at work in the same galaxy. Considering the spatial distributions of the dust brightness and temperature in the halo, the tidal interaction may be far more effective than the wind in removing the cool interstellar medium from the disk of M 82. We estimate that more than two thirds of the extraplanar dust mass is associated with the tidal streams traced by atomic hydrogen. If dust is able to survive for a long time in the extragalactic medium around such interacting systems, which may have been common in the past, then it will have significant column densities along lines of sight to distant galaxies, and cause extra attenuation outside the Milky Way.

Acknowledgements. We thank the referee for suggesting useful clarifications, L. Leeuw and I. Robson for providing their SCUBA images. SPIRE has been developed by a consortium of institutes led by Cardiff Univ. (UK): Univ. Lethbridge (Canada); NAOC (China); CEA, LAM (France); IFSI, Univ. Padua (Italy); IAC (Spain); Stockholm Obs. (Sweden); Imperial College London, RAL, UCLMSSL, UKATC, Univ. Sussex (UK); and Caltech, JPL, NHSC, Univ. Colorado (USA). This development has been supported by national funding agencies: CSA (Canada); NAOC (China); CEA, CNES, CNRS (France); ASI (Italy); MCINN (Spain); Stockholm Obs. (Sweden); STFC (UK); and NASA (USA).

\section{References}

Alton, P. B., Davies, J. I., \& Bianchi, S. 1999, A\&A, 343, 51 Boselli, A., \& Gavazzi, G. 2002, A\&A, 386, 124

Engelbracht, C. W., Kundurthy, P., Gordon, K. D., et al. 2006, ApJ, 642, L127 Förster Schreiber, N. M., Genzel, R., Lutz, D., et al. 2003, ApJ, 599, 193 Gallagher, J. S., \& Smith, L. J. 1999, MNRAS, 304, 540

Griffin, M. J., et al. 2010, A\&A, 518, L3

de Grijs, R., O'Connell, R. W., \& Gallagher, J. S., III 2001, AJ, 121, 768 Högbom, J. A. 1974, A\&AS, 15, 417

Hoopes, C. G., Heckman, T. M., Strickland, D. K., et al. 2005, ApJ, 619, L99 James, A., et al. 2003, MNRAS, 335, 753

Konstantopoulos, I. S., Bastian, N., Smith, L. J., et al. 2009, ApJ, 701, 1015 Leeuw, L. L., \& Robson, E. I. 2009, AJ, 137, 517

Li, A., \& Draine, B. T. 2001, ApJ, 554, 778

Mao, R. Q., Henkel, C., Schulz, A., et al. 2000, A\&A, 358, 433

Melo, V. P., Muñoz-Tuñón, C., et al. 2005, ApJ, 619, 270

Micelotta, E. R.,Jones, A. P., \& Tielens, A. G. G. M. 2010, A\&A, 510, A36

Muxlow, T. W., Pedlar, A., Wilkinson, P. N., et al. 1994, MNRAS, 266, 455

Panuzzo, P., et al. 2010, A\&A, 518, L37

Pilbratt, G. L., et al. 2010, A\&A, 518, L1

Pohlen, M., et al. 2010, A\&A, 518, L72

Reach, W. T., Dwek, E., Fixsen, D. J., et al. 1995, ApJ, 451, 188

Sakai, S., \& Madore, B. F. 1999, ApJ, 526, 599

Scarrott, S. M., Eaton, N., \& Axon, D. J. et al. 1991, MNRAS, 252, 12

Shopbell, P. L., \& Bland-Hawthorn, J. 1998, ApJ, 493, 129

Strickland, D. K., Ponman, T. J., Stevens, I. R., et al. 1997, A\&A, 320, 378

Telesco, C. M., \& Gezari, D. Y. 1992, ApJ, 395, 461

Thuma, G., et al. 2000, A\&A, 358, 65

Walter, F., Weiss, A., \& Scoville, N. 2002, ApJ, 580, L21

Ward, J. S., Zmuidzinas, J., Harris, A. I., \& Isaak, K. G. 2003, ApJ, 587, 171

Yun, M. S., Ho, P. T. P., \& Lo, K. Y. 1994, Nature, 372, 530

Page 5 is available in the electronic edition of the journal at http: //www . aanda. org 
1 Institut d'Astrophysique de Paris, UMR7095 CNRS, Université Pierre \& Marie Curie, 98 bis Boulevard Arago, 75014 Paris, France e-mail: roussel@iap.fr

2 Dept. of Physics \& Astronomy, McMaster University, Hamilton, Ontario, L8S 4M1, Canada

3 School of Physics \& Astronomy, Cardiff University, Queens Buildings The Parade, Cardiff CF24 3AA, UK

4 ESA Astrophysics Missions Division, ESTEC, PO Box 299, 2200 AG Noordwijk, The Netherlands

5 CEA, Laboratoire AIM, Irfu/SAp, Orme des Merisiers, 91191 Gifsur-Yvette, France

6 Sterrenkundig Observatorium, Universiteit Gent, Krijgslaan 281 S9, 9000 Gent, Belgium

7 Dept. of Physics \& Astronomy, University College London, Gower Street, London WC1E 6BT, UK

8 Astrophysics Group, Imperial College, Blackett Laboratory, Prince Consort Road, London SW7 2AZ, UK

9 Jet Propulsion Laboratory, Pasadena, CA 91109, USA; Dept. of Astronomy, California Institute of Technology, Pasadena, CA 91125, USA

10 Laboratoire d'Astrophysique de Marseille, UMR6110 CNRS, 38 rue F. Joliot-Curie, 13388 Marseille France

11 Instituto de Astrofísica de Canarias, vía Láctea S/N, 38200 La Laguna, Spain

12 Dept. of Physics \& Astronomy, University of California, Irvine, CA 92697, USA
13 Observational Cosmology Lab, Code 665, NASA Goddard Space Flight Center Greenbelt, MD 20771, USA

14 Dept. of Astrophysical \& Planetary Sciences, CASA CB-389, University of Colorado, Boulder, CO 80309, USA

15 Astronomy Centre, Dept. of Physics and Astronomy, University of Sussex, UK

16 Mullard Space Science Laboratory, University College London, Holmbury St Mary, Dorking, Surrey RH5 6NT, UK

17 School of Physics \& Astronomy, University of Nottingham, University Park, Nottingham NG7 2RD, UK

18 Istituto di Fisica dello Spazio Interplanetario, INAF, Via del Fosso del Cavaliere 100, 00133 Roma, Italy

19 Infrared Processing \& Analysis Center, California Institute of Technology, Mail Code 100-22, 770 South Wilson Av, Pasadena, CA 91125, USA

20 Centre for Astrophysics Research, Science \& Technology Research Centre, University of Hertfordshire, College Lane, Herts AL10 9AB, UK

21 University of Padova, Dept. of Astronomy, Vicolo Osservatorio 3, 35122 Padova, Italy

22 Observatoire Astronomique de Strasbourg, UMR 7550 Université de Strasbourg - CNRS, 11, rue de l'Université, 67000 Strasbourg, France

23 UK Astronomy Technology Center, Royal Observatory Edinburgh, Edinburgh, EH9 3HJ, UK

${ }^{24}$ Institut für Astronomie, Universität Wien, Türkenschanzstr. 17, 1180 Wien, Austria 